\title{
EIT-assisted atomic squeezing
}

\author{
A. Dantan ${ }^{1}$, M. Pinard ${ }^{1, a}$, and P.R. Berman ${ }^{2}$ \\ 1 Laboratoire Kastler Brossel, Case 74, 4 place Jussieu, 75252 Paris Cedex 05, France \\ 2 Michigan Center for Theoretical Physics, FOCUS Center, and Physics Department, University of Michigan, Ann Arbor, \\ Michigan 48109-1120, USA
}

Received 10 February 2003 / Received in final form 28 May 2003

Published online 12 August 2003 - (C) EDP Sciences, Società Italiana di Fisica, Springer-Verlag 2003

\begin{abstract}
The interaction of classical and quantized electromagnetic fields with an ensemble of atoms in an optical cavity is considered. Four fields drive a double- $\Lambda$ level scheme in the atoms, consisting of a pair of $\Lambda$ systems sharing the same set of lower levels. Two of the fields produce maximum coherence, $\rho_{12} \approx-1 / 2$, between the ground state sublevels 1 and 2 . This pumping scheme involves equal intensity fields that are resonant with both the one- and two-photon transitions of the $\Lambda$ system. There is no steady-state absorption of these fields, implying that the fields induce a type Electromagnetically-Induced Transparency (EIT) in the medium. An additional pair of fields interacting with the second $\Lambda$ system, combined with the EIT fields, leads to squeezing of the atom spin associated with the ground state sublevels. Our method involves a new mechanism for creating steady-state spin squeezing using an optical cavity. As the cooperativity parameter $C$ is increased, the optimal squeezing varies as $C^{-1 / 3}$. For experimentally accessible values of $C$, squeezing as large as $90 \%$ can be achieved.
\end{abstract}

PACS. 42.50.Lc Quantum fluctuations, quantum noise, and quantum jumps - 42.50.Dv Nonclassical states of the electromagnetic field, including entangled photon states; quantum state engineering and measurements - 42.65.Pc Optical bistability, multistability, and switching, including local field effects

\section{Introduction}

Spin squeezing refers to the reduction of noise in one of the components of the effective spin associated with an ensemble of two-level quantum systems. There has been a great deal of interest in spin squeezing as a means for reducing the quantum noise that is intrinsic to any precision measurement [1]. Several methods for achieving spin squeezing have been proposed [2-9], including a recent one of ours involving atoms interacting with a classical field and a quantized, cavity field in a $\Lambda$ configuration [10]. We have shown that, for a sufficiently large nonlinearity (large cooperativity parameter $C$ ), self-squeezing is obtained in such a 3-level atomic medium when the input field driving the cavity mode is a coherent state of the radiation field. Maximal squeezing occurs near the points of optical bistability in this system. With increasing cooperativity parameter $C$, the maximum self-squeezing that can be obtained is about $30 \%$. In this paper, we show that it is possible to increase this limit by modifying the pumping scheme. In effect, we introduce a method for pumping the coherence between the two ground state levels using a double- $\Lambda$ scheme [11].

The double- $\Lambda$ scheme consists of a pair of $\Lambda$ systems sharing the same set of lower levels. One of the $\Lambda$ schemes is designed to produce maximum coherence, $\rho_{12}=-1 / 2$, between the ground state levels 1 and 2 . This pumping scheme involves equal intensity fields that are resonant with both the one and two-photon transitions of the $\Lambda$ system and leads to pumping of the dark-state, $[(|1\rangle-|2\rangle) / \sqrt{2}]$. There is no steady-state absorption of these fields, implying that the fields induce a type of electromagnetically-induced transparency (EIT) $[12,13,15]$. The EIT fields' quantum correlations have been investigated in [14], but not the atom-field correlations. It is not difficult, however, to show that, if the input fields are in a coherent state, the spin associated with levels 1 and 2 resulting from these fields alone is at least as noisy that of a coherent spin state. Hence, EIT alone does not produce atomic spin squeezing. In order to squeeze the ground state atomic spin, we introduce an asymmetry in the system by considering an additional pair of fields interacting with the second $\Lambda$ system [10]. In the combined double- $\Lambda$ scheme, our calculations predict that there is no limit to the spin squeezing that can be achieved; the variance of one spin component approaches zero asymptotically as $C^{-1 / 3}$ for $C \gg 1$. We provide analytical calculations of the atomic variance, allowing one to optimize the squeezing and also discuss the process leading to the creation of squeezing, which is new and different from squeezing originating from optical bistability $[4,6]$. The EIT interaction enables one to pump the coherence

\footnotetext{
a e-mail: pinard@spectro.jussieu.fr
} 


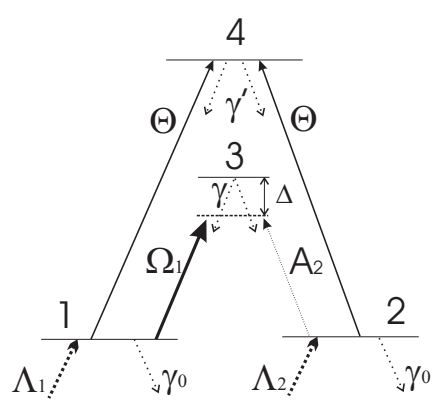

Fig. 1. Double- $\Lambda$ scheme.

and increase the spin mean value while the cavity coupling enhances the atomic cooperative behavior.

In Section 2, we describe the system and give the set of Heisenberg-Langevin equations governing the system. In Section 3, we provide an effective 2-level system, give analytical results for the optimal squeezing and discuss the squeezing creation process.

\section{Atom-field configuration}

The system considered in this paper consists of a set of $N$, 4-level atoms, whose levels form a double- $\Lambda$ configuration, as represented in Figure 1. On the lower $\Lambda$ transition $(1,3,2)$ the atoms interact with two light fields: an intense classical field $A_{1}$ in a single pass scheme on transition $1 \rightarrow 3$, and a quantum field $A_{2}$ in an optical cavity on the transition $2 \rightarrow 3$. The field frequencies are $\omega_{1}$ and $\omega_{2}$ and the detunings from atomic resonance $\Delta_{i}=\omega_{3 i}-\omega_{i}(i=1,2)$ are assumed to be much greater than the excited decay rate of state 3 . If $\omega_{c}$ is the cavity resonance frequency that is closest to the probe frequency, the cavity detuning for the quantum field can be defined as $\Delta_{c}=\omega_{2}-\omega_{c}$. An incoming quantum field $A_{2}^{\text {in }}$ drives the cavity field $A_{2}$. The field $A_{1}$ is treated classically and its intensity is supposed to be much greater than that of the quantum field. On the second $\Lambda$ transition, the atoms resonantly interact with the two modes of a quantum cavity field $\Theta$, with frequency $\omega^{\prime}$ and cavity detuning $\Delta_{c}^{\prime}$. Although both modes are represented by the same symbol $\Theta$, each mode drives only one transition, $1 \rightarrow 4$ or $2 \rightarrow 4$. This selectivity can be provided by field polarization if states 1 and 2 are degenerate, or by frequency selectivity if they belong to different ground state hyperfine manifolds.

The 4-level system is described using 16 collective operators for the $N$ atoms of the ensemble: the populations $\Pi_{i}=\sum_{\mu=1}^{N}|i\rangle_{\mu}\left\langle\left. i\right|_{\mu}(i=1-4)\right.$, the components of the optical dipoles $P_{i j}$ in the frames rotating at the frequency of their corresponding lasers and their Hermitian conjugates and the components of the dipole associated to the ground state coherence: $P_{r}=\sum_{\mu=1}^{N}|2\rangle_{\mu}\left\langle\left. 1\right|_{\mu}\right.$ and $P_{r}^{\dagger}$. We take $\omega_{1}=\omega_{2}$, so that the ground state coherence is excited at zero frequency in both $\Lambda$ s.
The coupling constant between atoms and field $A_{2}$ is defined by $g=\mathcal{E}_{0} d / \hbar$, where $d$ is the atomic dipole, $\mathcal{E}_{0}=\sqrt{\hbar \omega_{2} / 2 \epsilon_{0} \mathcal{S} c}$, and $\mathcal{S}$ is the cross-sectional area of the cavity field. With this definition, the mean square value of the field is expressed in number of photons per second. A second coupling constant $g^{\prime}$ is similarly defined for field $\Theta$. The decay constants of dipoles $P_{13}$ and $P_{23}$ are taken equal to $\gamma$, and those of $P_{14}$ and $P_{24}$ equal to $\gamma^{\prime}$. In order to take into account the finite lifetime of the two fundamental sublevels 1 and 2, we include in the model another decay rate $\gamma_{0}$, which is supposed to be much smaller than $\gamma$. For example $\gamma_{0}^{-1}$ can represent an atom's transit time in the light field, typically of the order of a few milliseconds for cold atoms. On the other hand, $\gamma$ and $\gamma^{\prime}$ are of the order of the $\mathrm{MHz}$ for excited states. We also consider that the sublevels 1 and 2 are repopulated with incoherent pumping terms $\Lambda_{1}$ and $\Lambda_{2}$, so that the total atomic population is kept constantly equal to $N$.

The system evolution is given by a set of quantum Heisenberg-Langevin equations

$$
\begin{aligned}
\frac{\mathrm{d} \Pi_{1}}{\mathrm{~d} t}= & \mathrm{i} \Omega_{1}^{*} P_{13}-\mathrm{i} \Omega_{1} P_{13}^{\dagger}+\mathrm{i} g^{\prime} \Theta^{\dagger} P_{14}-\mathrm{i} g^{\prime} \Theta P_{14}^{\dagger} \\
& +\gamma \Pi_{3}+\gamma^{\prime} \Pi_{4}-\gamma_{0} \Pi_{1}+\Lambda_{1}+F_{11} \\
\frac{\mathrm{d} \Pi_{2}}{\mathrm{~d} t}= & \mathrm{i} g A_{2}^{\dagger} P_{23}-\mathrm{i} g A_{2} P_{23}^{\dagger}+\mathrm{i} g^{\prime} \Theta^{\dagger} P_{24}-\mathrm{i} g^{\prime} \Theta P_{24}^{\dagger} \\
& +\gamma \Pi_{3}+\gamma^{\prime} \Pi_{4}-\gamma_{0} \Pi_{2}+\Lambda_{2}+F_{22} \\
\frac{\mathrm{d} \Pi_{3}}{\mathrm{~d} t}= & -\left(\mathrm{i} \Omega_{1}^{*} P_{13}-\mathrm{i} \Omega_{1} P_{13}^{\dagger}\right)-\left(\mathrm{i} g A_{2}^{\dagger} P_{23}-\mathrm{i} g A_{2} P_{23}^{\dagger}\right) \\
& -2 \gamma \Pi_{3}+F_{33} \\
\frac{\mathrm{d} \Pi_{4}}{\mathrm{~d} t}= & -\left(\mathrm{i} g^{\prime} \Theta^{\dagger} P_{14}-\mathrm{i} g^{\prime} \Theta P_{14}^{\dagger}\right)-\left(\mathrm{i} g^{\prime} \Theta^{\dagger} P_{24}-\mathrm{i} g^{\prime} \Theta P_{24}^{\dagger}\right) \\
& -2 \gamma^{\prime} \Pi_{4}+F_{44} \\
\frac{\mathrm{d} P_{13}}{\mathrm{~d} t}= & -\left(\gamma+\mathrm{i} \Delta_{1}\right) P_{13}+\mathrm{i} \Omega_{1}\left(\Pi_{1}-\Pi_{3}\right)+\mathrm{i} g A_{2} P_{r}^{\dagger}+F_{13}
\end{aligned}
$$

$\frac{\mathrm{d} P_{23}}{\mathrm{~d} t}=-\left(\gamma+\mathrm{i} \Delta_{2}\right) P_{23}+\mathrm{i} g A_{2}\left(\Pi_{2}-\Pi_{3}\right)+\mathrm{i} \Omega_{1} P_{r}+F_{23}$

$\frac{\mathrm{d} P_{14}}{\mathrm{~d} t}=-\gamma^{\prime} P_{14}+\mathrm{i} g^{\prime} \Theta\left(\Pi_{1}-\Pi_{4}\right)+\mathrm{i} g^{\prime} \Theta P_{r}^{\dagger}+F_{14}$

$\frac{\mathrm{d} P_{24}}{\mathrm{~d} t}=-\gamma^{\prime} P_{24}+\mathrm{i} g^{\prime} \Theta\left(\Pi_{2}-\Pi_{4}\right)+\mathrm{i} g^{\prime} \Theta P_{r}+F_{24}$

$\frac{\mathrm{d} P_{r}}{\mathrm{~d} t}=-\left(\gamma_{0}-\mathrm{i} \delta\right) P_{r}+\mathrm{i} \Omega_{1}^{*} P_{23}-\mathrm{i} g A_{2} P_{13}^{\dagger}$

$+\mathrm{i} g^{\prime} \Theta^{\dagger} P_{24}-\mathrm{i} g^{\prime} \Theta P_{14}^{\dagger}+F_{21}$
$\frac{\mathrm{d} A_{2}}{\mathrm{~d} t}=-\left(\kappa+\mathrm{i} \Delta_{c}\right) A_{2}+\frac{\mathrm{i} g}{\tau} P_{23}+\sqrt{\frac{2 \kappa}{\tau}} A_{2}^{\mathrm{in}}$
$\frac{\mathrm{d} \Theta}{\mathrm{d} t}=-\left(\kappa^{\prime}+\mathrm{i} \Delta_{c}^{\prime}\right) \Theta+\frac{\mathrm{i} g^{\prime}}{\tau^{\prime}}\left(P_{14}+P_{24}\right)+\sqrt{\frac{2 \kappa^{\prime}}{\tau^{\prime}}} \Theta^{\mathrm{in}}$

where $g$ and $g^{\prime}$ are assumed real, $\Omega_{1}=g A_{1}, \delta=\Delta_{1}-\Delta_{2}$ is the frequency difference between the ground state sublevels, $\kappa$ and $\kappa^{\prime}$ are the intracavity field decays and $\tau$ and $\tau^{\prime}$ are the round trip times in the cavity. The EIT fields are 
taken to be quantum cavity fields in the bad cavity limit $\left(\gamma^{\prime} \ll \kappa^{\prime}\right)$, but could just as well have been taken to be classical fields propagating in free space. From the previous set of equations, it is possible to derive the steady state values and the correlation matrix for the fluctuations of the atom-field system (see e.g. [4]). Our aim here is to obtain the fluctuations of the spin operators associated with levels 1 and 2 from simplified equations for the ground state variables as in reference [10].

\section{Simplified equations for the ground state observables}

Owing to the off-resonant interaction on transitions $1 \rightarrow 3$ and $2 \rightarrow 3$, the excited state population $\left\langle\Pi_{3}\right\rangle$ is negligible and the optical coherences $P_{13}$ and $P_{23}$ evolve rapidly compared to $\Pi_{1}, \Pi_{2}$ and $P_{r}$. It is also reasonable to assume that $\delta=\Delta_{1}-\Delta_{2} \ll \Delta=\left(\Delta_{1}+\Delta_{2}\right) / 2$. On transitions $1 \rightarrow 4$ and $2 \rightarrow 4$ we choose a pumping rate $\Gamma_{p}^{\prime}=2 g^{\prime 2}|\langle\Theta\rangle|^{2} / \gamma^{\prime}$ much smaller than $\gamma^{\prime}$ so that $\left\langle\Pi_{4}\right\rangle$ is negligible and the optical coherences $P_{14}$ and $P_{24}$ adiabatically follow the ground state observables. Eliminating the excited state populations and replacing the optical coherences in equations (1-11) by their steady state values, one gets simplified equations for the ground state variables $S_{+}=P_{r}, S_{-}=P_{r}^{\dagger}, S_{z}=\left(\Pi_{2}-\Pi_{1}\right) / 2$ and the quantum field $A_{2}$,

$$
\begin{aligned}
& \frac{\mathrm{d} S_{+}}{\mathrm{d} t}=-\left(\tilde{\gamma}_{0}-\mathrm{i} \tilde{\delta}\right) S_{+}+\tilde{\Lambda}_{12}+2 \mathrm{i} \tilde{g} A_{2} S_{z}+F_{+} \\
& \frac{\mathrm{d} S_{z}}{\mathrm{~d} t}=-\tilde{\gamma}_{0} S_{z}+\frac{\tilde{\Lambda}_{2}-\tilde{\Lambda}_{1}}{2}+\mathrm{i} \tilde{g}\left(A_{2}^{\dagger} S_{+}-S_{-} A_{2}\right)+F_{z} \\
& \frac{\mathrm{d} A_{2}}{\mathrm{~d} t}=-\left(\kappa+\mathrm{i} \Delta_{c}\right) A_{2}+\mathrm{i} \frac{\tilde{g}}{\tau} S_{+}+\sqrt{\frac{2 \kappa}{\tau}} A_{2}^{\mathrm{in}}
\end{aligned}
$$

where $\tilde{\delta}=\delta+\left|\Omega_{1}^{2}\right| / \Delta$ is the effective atomic detuning corrected with the light-shift and $\tilde{g}=g \Omega_{1} / \Delta$ is the effective coupling constant. Denoting by $\Gamma_{p}=\gamma\left|\Omega_{1}^{2}\right| / \Delta^{2}$ the optical pumping rate due to field $A_{1}$, the new in-terms and decay constants are then

$$
\begin{aligned}
\tilde{\gamma}_{0} & =\gamma_{0}+\Gamma_{p}+\Gamma_{p}^{\prime} \\
\tilde{\Lambda}_{2}-\tilde{\Lambda}_{1} & =\Lambda_{2}-\Lambda_{1}+N \Gamma_{p} \\
\tilde{\Lambda}_{12} & =-N \Gamma_{p}^{\prime} / 2 .
\end{aligned}
$$

We assume a symmetrical configuration $\left(\Lambda_{1}=\Lambda_{2}\right)$ and that the population is constant $\left(\Lambda_{1}+\Lambda_{2}=N \gamma_{0}\right)$. To get (14), we have used the fact that $\left|\Omega_{1}^{2}\right| \gg\left|g^{2}\left\langle A_{2}^{\dagger} A_{2}\right\rangle\right|$. This effective system is now quite similar to that of reference [10] in the case of the single $\Lambda$ Raman interaction, the essential difference is that EIT or dark-state pumping results in an in-term for the ground state coherence. As usual, we introduce the parameter $C$ quantifying the cooperative behavior of the atomic ensemble

$$
C=\frac{g^{2} N}{2 \kappa \tau \gamma} \text {. }
$$

The steady-state can be obtained setting the time derivatives to 0 in equations $(12-14)$ and using the fact that the Langevin operators mean values are 0 . Since we are interested in the quantum fluctuations, we linearize the effective equations around their steady-state values, assuming fluctuations are small with respect to mean values.

\subsection{Linearization and diffusion matrix}

The linearized equations for the fluctuations may be written in a matrix form

$$
\left.\left.\frac{\mathrm{d} \mid \delta \xi(t)]}{\mathrm{d} t}=-[B] \mid \delta \xi(t)\right]+\mid F_{\xi}\right]
$$

where $\mid \delta \xi(t)]=\left[\delta A_{2}(t), \delta A_{2}^{\dagger}(t), \delta S_{+}(t), \delta S_{-}(t),\left.\delta S_{z}(t)\right|^{T}\right.$ is the fluctuation vector, $[B]$ is the linearized evolution matrix

$$
[B]=\left(\begin{array}{ccccc}
\kappa+\mathrm{i} \Delta_{c} & 0 & -\mathrm{i} \tilde{g} / \tau & 0 & 0 \\
0 & \kappa-\mathrm{i} \Delta_{c} & 0 & \mathrm{i} \tilde{g} / \tau & 0 \\
-2 \mathrm{i} \tilde{g}\left\langle S_{z}\right\rangle & 0 & \tilde{\gamma}_{0}-\mathrm{i} \tilde{\delta} & 0 & -2 \mathrm{i} \tilde{g}\left\langle A_{2}\right\rangle \\
0 & 2 \mathrm{i} \tilde{g}\left\langle S_{z}\right\rangle & 0 & \tilde{\gamma}_{0}+\mathrm{i} \tilde{\delta} & 2 \mathrm{i} \tilde{g}\left\langle A_{2}\right\rangle^{*} \\
\mathrm{i} \tilde{g}\left\langle S_{-}\right\rangle & -\mathrm{i} \tilde{g}\left\langle S_{+}\right\rangle & -\mathrm{i} \tilde{g}\left\langle A_{2}\right\rangle^{*} \mathrm{i} \tilde{g}\left\langle A_{2}\right\rangle & \tilde{\gamma}_{0}
\end{array}\right)
$$

and $\left.\mid F_{\xi}\right]$ is the column vector regrouping the corresponding Langevin operators. As in [4], we define the covariance matrix $[G(t)]$ by

$$
[G(t)]=\mid \delta \xi(t)][\delta \xi(0) \mid
$$

and the diffusion matrix by

$$
\left.\mid F_{\xi}(t)\right]\left[F_{\xi}\left(t^{\prime}\right) \mid=[D] \delta\left(t-t^{\prime}\right) .\right.
$$

The values of the atomic diffusion coefficients can be derived from the quantum regression theorem [16]. The complete diffusion matrix is given in Appendix. The variances of the spin components and their correlation functions are the elements of the zero time correlation matrix $[G(0)]$, which satisfies [17]

$$
[B][G(0)]+[G(0)][B]^{\dagger}=[D] .
$$

The inverse of equation (15) gives $[G(0)]$, and, consequently, the spin variances. We then proceed with the calculation of the minimal variance in the plane orthogonal to the mean spin as in $[4,10]$.

\subsection{Optimal squeezing for $\left\langle\mathbf{A}_{2}\right\rangle=0$}

From the numerical calculations outlined in Section 3.1, the optimal atomic squeezing was found to occur at twophoton resonance $(\tilde{\delta}=0)$ and when the quantum cavity field mean value was 0 , with no cavity detuning $\left(\tilde{\Delta}_{c}=0\right)$. The effect of a non-zero mean value of the cavity field will be discussed later. In particular, we will show that the 
results obtained in this section still hold for small values of the quantum field intensity. Since the calculations are much easier and the physical meaning quite clear, we focus on the case $\left\langle A_{2}\right\rangle=0$. The steady state is then simple: $\left\langle S_{z}\right\rangle=N \Gamma_{p} / 2 \tilde{\gamma}_{0}$ and $\left\langle S_{+}\right\rangle=\left\langle S_{-}\right\rangle=-N \Gamma_{p}^{\prime} / 2 \tilde{\gamma}_{0}$. Rewriting the equations for atomic fluctuations in the $S_{x}, S_{y}, S_{z}$ basis and defining the usual quadrature operators for $A_{2}$,

$$
E_{P}=\frac{A_{2}+A_{2}^{\dagger}}{2}, \quad E_{Q}=\frac{A_{2}-A_{2}^{\dagger}}{2 \mathrm{i}}
$$

one obtains the following set of equations

$$
\begin{aligned}
\frac{\mathrm{d}}{\mathrm{d} t} \delta S_{x} & =-\tilde{\gamma}_{0} \delta S_{x}-2 \tilde{g}\left\langle S_{z}\right\rangle \delta E_{Q}+F_{x} \\
\frac{\mathrm{d}}{\mathrm{d} t} \delta S_{y} & =-\tilde{\gamma}_{0} \delta S_{y}+2 \tilde{g}\left\langle S_{z}\right\rangle \delta E_{P}+F_{y} \\
\frac{\mathrm{d}}{\mathrm{d} t} \delta S_{z} & =-\tilde{\gamma}_{0} \delta S_{z}-2 \tilde{g}\left\langle S_{+}\right\rangle \delta E_{Q}+F_{z} \\
\frac{\mathrm{d}}{\mathrm{d} t} \delta E_{P} & =-\kappa \delta E_{P}-\frac{\tilde{g}}{\tau} \delta S_{y}+\sqrt{\frac{2 \kappa}{\tau}} \delta E_{P}^{\mathrm{in}} \\
\frac{\mathrm{d}}{\mathrm{d} t} \delta E_{Q} & =-\kappa \delta E_{Q}+\frac{\tilde{g}}{\tau} \delta S_{x}+\sqrt{\frac{2 \kappa}{\tau}} \delta E_{Q}^{\mathrm{in}} .
\end{aligned}
$$

It can be seen that $\delta S_{y}$ is coupled only to $\delta E_{P}$, while $\delta S_{x}$ and $\delta S_{z}$ are coupled together via $\delta E_{Q}$. By Fouriertransforming and integrating the linear set of equations, one can show that the minimal variance is that of the $y$-component of the spin, which is unchanged when one transforms to a basis where the $z$-axis is aligned along the mean spin. Indeed, since the mean spin is always in the $x, z$-plane, the $y$-component is orthogonal to the mean spin, whereas the noisiest component in the plane orthogonal to the mean spin is a linear combination of $\delta S_{z}$ and $\delta S_{x}$. The relevant component for squeezing is therefore $S_{y}$, the variance of which is

$$
\left\langle\delta S_{y}^{2}\right\rangle=\frac{N}{4}\left[1-\frac{2 C}{1+\tilde{\rho}} \frac{\Gamma_{p}^{2}\left(\gamma_{0}+\Gamma_{p}^{\prime}\right)}{\tilde{\gamma}_{0}\left(\tilde{\gamma}_{0}^{2}+2 C \Gamma_{p}^{2}\right)}\right]
$$

in which $\tilde{\rho}=\tilde{\gamma}_{0} / \kappa=\rho \tilde{\gamma}_{0} / \gamma_{0}$ is the ratio of atomic and field decay rate and $\rho=\gamma_{0} / \kappa$. Equation (21) clearly shows that the fluctuations are small when $\tilde{\rho} \ll 1$ (bad-cavity limit) and $C \gg 1$ (high cooperative behavior). The criterion for spin squeezing is obtained comparing the minimal variance to half the spin mean value [19],

$$
|\langle\mathbf{S}\rangle| / 2=N \frac{\sqrt{\Gamma_{p}^{\prime 2}+\Gamma_{p}^{2}}}{4 \tilde{\gamma}_{0}} .
$$

Consistent with the definition given in [19] and with our previous work $[4-6,10]$, the atoms are said to be squeezed when

$$
\Delta S_{\min }=\frac{\left\langle\delta S_{y}^{2}\right\rangle}{|\langle\mathbf{S}\rangle| / 2}<1 .
$$

In Figure 2, we plot the minimum variance as a function of the EIT pumping rate: $\Delta S_{\text {min }}$ goes through a minimum in the range of pump strengths satisfying $\gamma_{0} \ll \Gamma_{p}^{\prime} \ll \gamma^{\prime}$. The

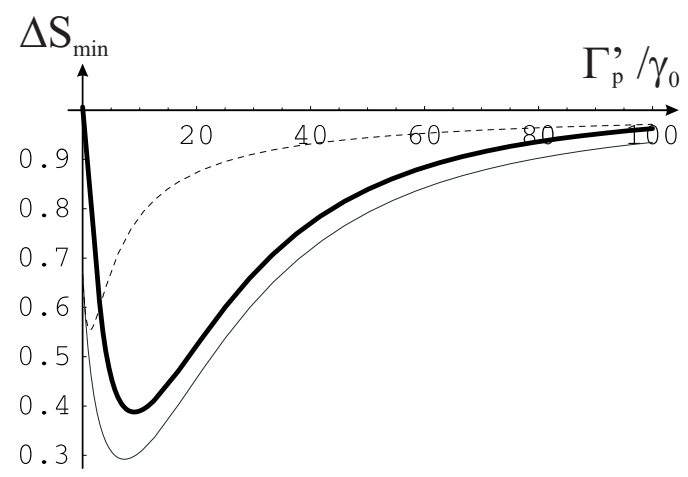

Fig. 2. Minimal variance $\Delta S_{\min }$ [thick line] versus EIT pumping rate $\Gamma_{p}^{\prime}$, expressed in $\gamma_{0}$ units. The mean spin half value $|\langle\mathbf{S}\rangle| / 2$ [dashed line] and the minimal spin fluctuations $\left\langle\delta S_{y}^{2}\right\rangle$ [thin line], normalized by $N / 4$, are also represented for the same parameters $\left(C=100, \rho=1 / 2000, \Gamma_{p}=2 \gamma_{0}\right)$.

fluctuations of (21) should be compared to the mean spin half value $|\langle\mathbf{S}\rangle| / 2$ of (22). These two quantities, normalized by $N / 4$, are plotted versus the EIT pumping rate in Figure 2 for given values of $C, \tilde{\rho}$ and $\Gamma_{p}$. Both go through a minimum with increasing $\Gamma_{p}^{\prime}$, but there exists a regime in which the spin mean value is increased more than the fluctuations. In this regime, the atom spin is squeezed. Note that when the EIT interaction is absent $\left(\Gamma_{p}^{\prime}=0\right)$, as well as when it is predominant $\left(\Gamma_{p}^{\prime} \sim 100 \gamma_{0}\right)$, there is little or no squeezing. However, for intermediate values of the pumping rate, the EIT interaction allows one to pump the mean spin while the fluctuations are close to their minimal value. The best squeezing for a fixed value of $\Gamma_{p}$ is determined by the biggest "gap" between $\left\langle\delta S_{y}^{2}\right\rangle$ and $|\langle\mathbf{S}\rangle| / 2$. The qualitative dependence of spin squeezing can be understood as follows: one must have $\Gamma_{p}^{\prime} \gg \gamma_{0}$ to produce significant pumping of the coherence $\rho_{12}$; however, the entanglement of the spins with the cooperativity parameter must be sufficiently large to dominate the fluctuations produced by the pumping fields. As can be deduced from equation (21), the condition on the cooperativity parameter translates into the condition $\Gamma_{p}^{\prime} \ll \kappa$ and $C \Gamma_{p}^{2} / \tilde{\gamma}_{0}^{2}=$ $C \Gamma_{p}^{2} /\left(\gamma_{0}+\Gamma_{p}+\Gamma_{p}^{\prime}\right)^{2} \geq 1$. Both this inequality and the condition that $\tilde{\rho}=\rho \tilde{\gamma}_{0} / \gamma_{0}=\left(\gamma_{0}+\Gamma_{p}+\Gamma_{p}^{\prime}\right) / \kappa \ll 1$ are violated for sufficiently large $\Gamma_{p}^{\prime}$. Note also that for $\Gamma_{p}=0$ (EIT interaction alone), the fluctuations are $N / 4$, and $\Delta S_{\min }=1+\gamma_{0} / \Gamma_{p}^{\prime} \geq 1$ confirming our statement that EIT alone does not produce atomic squeezing, but leaves the atoms in a more noisy state than a coherent spin state.

\subsection{Optimized variance}

The two pumping rates $\Gamma_{p}^{\prime}$ and $\Gamma_{p}$ can be optimized in order to minimize $\Delta S_{\min }$. The optimal values of $\Gamma_{p}^{\prime}$ and $\Gamma_{p}$, denoted by $\Gamma_{p}^{*}$ and $\Gamma_{p}^{*}$, can be obtained easily from the minima in Figure 3, which shows $\Delta S_{\min }$ versus $\Gamma_{p}^{\prime}$ for different values of $\Gamma_{p}$. However, in order to better understand the behavior of the variance with the cooperativity parameter, one can find approximate expressions for $\Gamma_{p}^{\prime *}$ and $\Gamma_{p}^{*}$ 


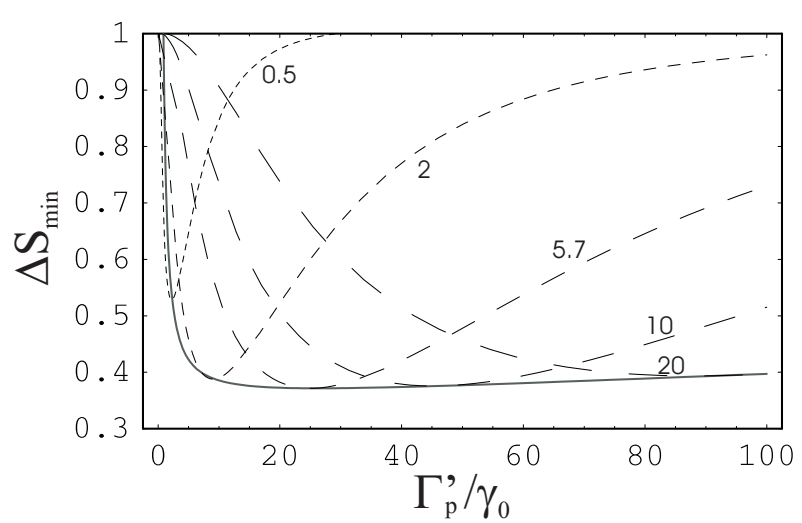

Fig. 3. Minimal variance $\Delta S_{\min }$ versus $\Gamma_{p}^{\prime}$ (in $\gamma_{0}$ units), for various values of the Raman pumping rate $\Gamma_{p} / \gamma_{0}=$ $0.5,2,5.7,10,20$ [dash spacing increases with $\Gamma_{p}$ ]. $C$ and $\rho$ are equal to 100 and $1 / 2000$. The plain curve shows the minimal variance in which $\Gamma_{p}=\Gamma_{p}^{*}=C^{-1 / 3} \Gamma_{p}^{\prime}$ was optimized as in (25). The optimal squeezing is found to occur for $\Gamma_{p}^{*}=5.5 \gamma_{0}$ and $\Gamma_{p}^{*}=25 \gamma_{0}$.

in the regime of interest $C \gg 1$ and $\rho=\gamma_{0} / \kappa \ll 1$ [typical experimental values are $C \sim 100-1000, \rho \sim 1 / 2000]$. Explicitly, if $C \gg 1$, but

$$
\rho C^{1 / 3} \ll 1
$$

one obtains from equation (23)

$$
\Gamma_{p}^{*} \simeq \sqrt{\frac{3}{2 \rho C}} \gamma_{0}, \quad \Gamma_{p}^{\prime *} \simeq \sqrt{\frac{3}{2 \rho C^{1 / 3}}} \gamma_{0}
$$

and

$$
\Delta S_{\min }^{*} \sim \frac{3}{2 C^{1 / 3}}\left(1+\frac{1}{2 C^{1 / 3}}+\frac{2 \sqrt{6 \rho C^{1 / 3}}}{3}\right) .
$$

In this limit, the minimum squeezing approaches zero as $C^{-1 / 3}$ with increasing $C$, the contribution of the last term in equation (26) being very small. In Figure 4, we plot equation (26), approximated by $\Delta S_{\min }^{*} \simeq 1.74 / C^{1 / 3}$ over the range of $C$ considered, to show that it agrees very well with the exact results.

For equation (26) to be valid, $\Gamma_{p}^{\prime}$ and $\Gamma_{p}$ have to be smaller than $\gamma^{\prime}$ and $\gamma$ for the adiabatic eliminations to be justified. The optimized value of $\Gamma_{p}$ is typically of the order of a few $\gamma_{0}$, so that the validity of our treatment is almost always ensured under the optimized conditions: $\Gamma_{p} \sim \gamma_{0}$ and $\gamma_{0} \ll \Gamma_{p}^{\prime} \ll \gamma^{\prime}$. The validity of the approximations was checked with a full 4-level calculation, the principle of which has been described in references $[4,6]$. We checked, in particular, that the noise coming from the quantum fluctuations of the EIT pumping fields is negligible in the regime of interest. It should be noted that the convergence of $\Delta S_{\min }^{*}$ to 0 with $C$ is rather slow because of the exponent $1 / 3$; increasing the atoms by a factor $10 \mathrm{im}-$ proves the squeezing by about $3 \mathrm{~dB}$. However, very good squeezing values can be obtained for standard experimental conditions. In Figure 4 the optimal squeezing in $\mathrm{dB}$ is

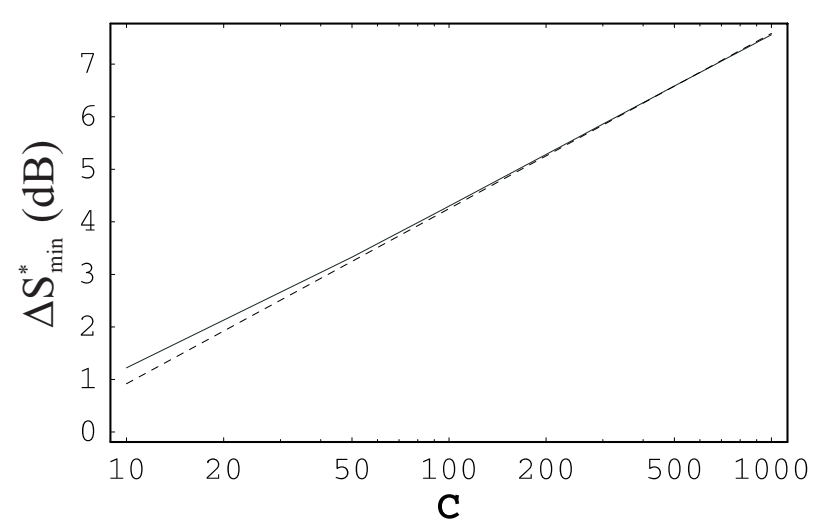

Fig. 4. Optimal squeezing (in $\mathrm{dB}$ ) versus the cooperativity $C$ on a log-log scale $(\rho=1 / 2000)$, from analytical expression [solid line] and fit [dashed line] given by (26).

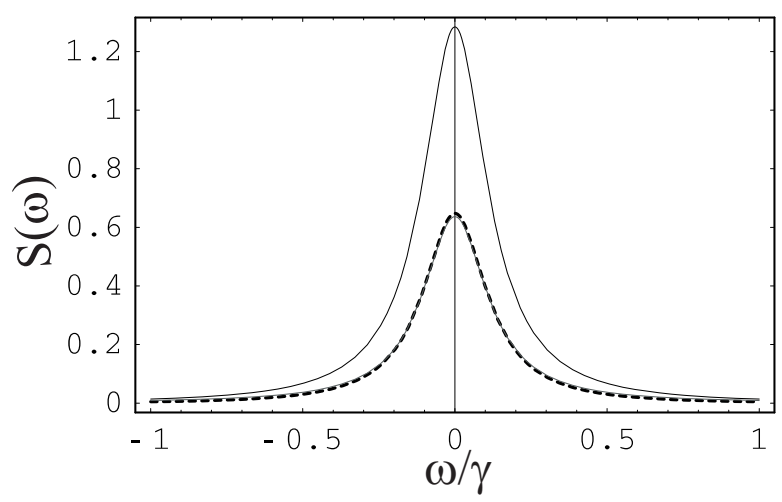

Fig. 5. Contributions to the atomic noise spectrum (normalized to $N$ ) [upper solid curve]: the contribution of the fluctuations of the incident quantum field $S_{f}$ [lower solid curve] is of the same order of $S_{\text {at }}$ [dashed curve]. The spectrum width is $2 \gamma_{+} \simeq 455 \gamma_{0}$ in the case considered $\left(C=100, \gamma=1000 \gamma_{0}\right.$, $\left.\rho=1 / 2000, \Gamma_{p}^{*}=5.5 \gamma_{0}, \Gamma_{p}^{\prime *}=25 \gamma_{0}\right)$.

plotted versus $C$. For $C=100$, we get $63 \%(4.3 \mathrm{~dB})$ of squeezing. Increasing the cooperativity to 1000 [18] would allow squeezing values of $83 \%$ (7.7 dB).

\subsection{Contributions to the atomic noise spectrum}

As in reference [10], it is interesting to go into the Fourier domain from (16-20) and plot the contributions to the atomic spectrum. The atomic spectrum of the minimal component, $\left\langle\delta S_{y}^{2}(\bar{\omega})\right\rangle$, is the sum of the incident field fluctuations $S_{f}$ and the atomic noise $S_{\text {at }}$ :

$$
S_{f}=\frac{N C \Gamma_{p}^{3}}{\kappa \tilde{\gamma}_{0}^{2}} \frac{1}{|D(\bar{\omega})|^{2}} ; \quad S_{\text {at }}=\frac{N \tilde{\rho}}{2} \frac{1+\bar{\omega}^{2}}{|D(\bar{\omega})|^{2}}
$$

where $D(\bar{\omega})=(1-\mathrm{i} \bar{\omega})(\tilde{\rho}-\mathrm{i} \bar{\omega})+2 \rho C \Gamma_{p}^{2} / \gamma_{0} \tilde{\gamma}_{0}$ and $\bar{\omega}=\omega / \kappa$. These contributions are represented in Figure 5 for optimized pumping values. Unlike the spectra derived in [10], we see that in the present case both contributions are of the same order of magnitude. The frequency width of the spectra can be found by noticing that, if we are in the 


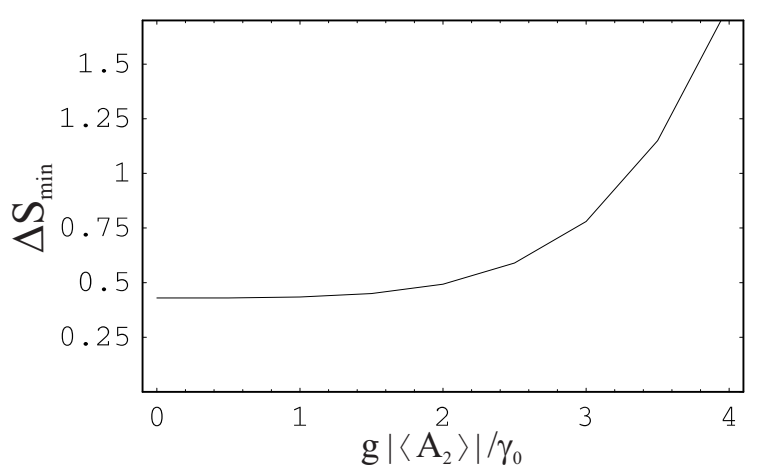

Fig. 6. Minimal variance $\Delta S_{\min }$ versus intracavity field amplitude $\left|\tilde{g}\left\langle A_{2}\right\rangle / \gamma_{0}\right|$. The parameters are $C=100, \rho=1 / 2000$, $\Gamma_{p}=\gamma_{0}, \Gamma_{p}^{\prime}=4 \gamma_{0}$. The field amplitude limit as calculated from Section 3.5 is $1.1 \gamma_{0}$ in this case.

bad-cavity limit: $\kappa \gg \tilde{\gamma}_{0}$, and one eliminates the field fluctuations in (19) and rewrites (17), the effective time constant for $\delta S_{y}$ is $\gamma_{+}=\tilde{\gamma}_{0}+2 C \Gamma_{p}^{2} / \tilde{\gamma}_{0}$, which can be made much greater than $\tilde{\gamma}_{0}$ [as in Fig. 5].

\subsection{Variation with the quantum cavity field intensity}

If the cavity field mean value is non-zero, the fluctuations of $S_{y}$ are coupled to both $E_{P}$ and $E_{Q}$ and equation (17) has an additional term proportional to $\left\langle A_{2}\right\rangle \delta S_{z}$. As a consequence, $\delta S_{y}$ is coupled to the other spin components. The minimal component in the plane orthogonal to the mean spin is then shifted, and the shift increases when $\left|\left\langle A_{2}\right\rangle\right|$ increases and its fluctuations are greater. Figure 6 shows the minimal variance versus the cavity field mean value for a given set of parameters. The squeezing is indeed destroyed when the field amplitude becomes too large. Yet, there exists a substantial range of intracavity intensities that do not destroy the squeezing too much. A lower limit for the field amplitude is set by looking at the linearized equation for $\delta S_{y}$, in which the field mean value is non zero and the field fluctuations have been adiabatically eliminated

$$
\frac{\mathrm{d}}{\mathrm{d} t} \delta S_{y}=-\gamma_{+} \delta S_{y}+2 \tilde{g}\left\langle S_{z}\right\rangle \delta E_{P}+2 \tilde{g}\left\langle A_{2}\right\rangle \delta S_{z}+F_{y}^{\prime} .
$$

From the previous equation, one may expect squeezing to be degraded when $\gamma_{+}^{2}\left\langle\delta S_{y}^{2}\right\rangle \sim 4\left(\tilde{g}\left\langle A_{2}\right\rangle\right)^{2}\left\langle\delta S_{z}^{2}\right\rangle$. Taking the analytical expressions for the variances (not reproduced here for simplicity) and assuming again that the incident field has standard quantum fluctuations, one thus gets a limiting value for the field amplitude $\left|\tilde{g}\left\langle A_{2}\right\rangle\right|$. The calculations of Section 3.2 for $\left\langle A_{2}\right\rangle=0$ are valid at least up to this limit, as can be seen from Figure 6 .

\section{Conclusion}

In $[4,6,10]$, the squeezing arose from the non-linear interaction between the fields and atoms in the vicinity of the lower turning point of the bistability curve. The atom- field system exhibits the analog of a first-order phase transition and that quantum fluctuations are important near the bistable point, allowing for either the field or the atoms to be squeezed. The critical parameter governing the behavior is the cooperativity parameter $C$. The underlying mechanism responsible for spin squeezing considered in this paper is rather different from bistability squeezing. Rather than originating in a regime where the amplitude of the fluctuations is either big or small, the squeezing can be traced to a region of parameter space where the fluctuations can be made small owing to the cooperative behavior of the atoms due to the cavity coupling. At the same time, the spin mean value increases faster than the fluctuations with increasing EIT pumping rate. We can then say that the spin is pumped owing to the EIT interaction, while the fluctuations are kept low by the cavity coupling. The consequence of this novel effect is that the atomic squeezing no longer saturates at some constant value when one increases the number of atoms. We would like to point out that the origin of squeezing is rather complex, in the sense that both the cavity coupling and the EIT pumping are necessary to produce squeezing, although each scheme, taken alone, does not yield squeezing; hence our appellation of EIT-assisted atomic squeezing. Note that the squeezing can be easily controlled via the EIT intensity, and the optimization provided by the rather simple analytical results of the effective system. In addition to the advantages of a cw experiment, we would like to point out that the long life-time of the ground state should render easier the squeezing detection and control.

The work of PRB was supported by the National Science Foundation under Grant No. PHY-0098016 and the FOCUS Center grant, and by the U.S. Army Research Office under Grant No. DAAD19-00-1-0412.

\section{Appendix}

We give the expression of the atomic diffusion coefficients in the case discussed in Section 3.1, when $\left\langle A_{2}\right\rangle=0, \tilde{\delta}=0$, $\Delta_{c}=0$. They were evaluated with the Einstein generalized relations [16] and grouped in the atomic diffusion matrix $\left[D_{\text {at }}\right]$

$$
\begin{aligned}
& {\left[D_{\mathrm{at}}\right]=} \\
& N\left[\begin{array}{ccc}
\tilde{\gamma}_{0}+\Gamma_{p}-\frac{\Gamma_{p}^{\prime 2}}{2 \tilde{\gamma}_{0}} & -\frac{\Gamma_{p}^{\prime 2}}{2 \tilde{\gamma}_{0}} & \left(\tilde{\gamma}_{0}+\Gamma_{p}\right) \frac{\Gamma_{p}^{\prime}}{2 \tilde{\gamma}_{0}} \\
-\frac{\Gamma_{p}^{\prime 2}}{2 \tilde{\gamma}_{0}} & \tilde{\gamma}_{0}-\Gamma_{p}-\frac{\Gamma_{p}^{\prime 2}}{2 \tilde{\gamma}_{0}} & \left(-\tilde{\gamma}_{0}+\Gamma_{p}\right) \frac{\Gamma_{p}^{\prime}}{2 \tilde{\gamma}_{0}} \\
\left(\tilde{\gamma}_{0}+\Gamma_{p}\right) \frac{\Gamma_{p}^{\prime}}{2 \tilde{\gamma}_{0}} & \left(-\tilde{\gamma}_{0}+\Gamma_{p}\right) \frac{\Gamma_{p}^{\prime}}{2 \tilde{\gamma}_{0}} & \frac{\tilde{\gamma}_{0}}{2}-\frac{\Gamma_{p}^{\prime 2}}{2 \tilde{\gamma}_{0}}
\end{array}\right] .
\end{aligned}
$$

\section{References}

1. D.J. Wineland, J.J. Bollinger, W.M. Itano, D.J. Heinzen, Phys. Rev. A 50, 67 (1994) 
2. A. Kuzmich, K. Molmer, E.S. Polzik, Phys. Rev. Lett. 79, 4782 (1997); J. Hald, J.L. Soerensen, C. Schori, E.S. Polzik, Phys. Rev. Lett. 83, 1319 (1999)

3. A. Kuzmich, L. Mandel, J. Janis, Y.E. Young, R. Ejnisman, N.P. Bigelow, Phys. Rev. A 60, 2346 (1999).

4. L. Vernac, M. Pinard, E. Giacobino, Phys. Rev. A 62, $063812(2000)$

5. L. Vernac, M. Pinard, E. Giacobino, Eur. Phys. J. D 17, $125(2001)$

6. L. Vernac, M. Pinard, V. Josse, E. Giacobino, Eur. Phys. J. D 18, 129 (2002)

7. I. Bouchoule, K. Molmer, Phys. Rev. A 65, 041803 (2002)

8. A. Andre, M.D. Lukin, Phys. Rev. A 65, 053819 (2002)

9. I. Bouchoule, K. Molmer, Phys. Rev. A 66, 043811 (2002)

10. A. Dantan, M. Pinard, V. Josse, N. Nayak, P.R. Berman, Phys. Rev. A 67, 045801 (2003)

11. For a review of double-lambda sytems with additional references, see M. Lukin, P.R. Hemmer, M.O. Scully, in Advances in Optical, Molecular and Optical Physics, edited by B. Bederson, H. Walther (Academic Press, San Diego, 2000), Vol. 42, pp. 347-386; see also A.J. Merriam, S.J. Sharpe, M. Shverdin, D. Manuszak, G.Y. Yin, S.E. Harris, Phys. Rev. Lett. 84, 5308 (2000); for a study of field fluctuations in a double- $\Lambda$ system, see E.A. Korsunsky, D.V. Kosachiov, Phys. Rev. A 60, 4996 (1999)
12. S.E. Harris, L.V. Hau, Phys. Rev. Lett. 82, 4611 (1999); C. Liu, Z. Dutton, C.H. Behroozi, L.V. Hau, Nature 409, $490(2001)$

13. D.F. Phillips, A. Fleischhauer, A. Mair, R.L. Walsworth, M.D. Lukin, Phys. Rev. Lett. 86, 783 (2001)

14. C.L. Garrido-Alzar, L.S. Cruz, J.G. Aguirre Gómez, M. França Santos, P. Nussenzveig, Europhys. Lett. 61, 485 (2003); C.L. Garrido-Alzar, M. França Santos, P. Nussenzveig, quant-ph/0205119

15. O. Kocharovskaya, Y. Rostovstev, M.O. Scully, Phys. Rev. Lett. 86, 628 (2001)

16. C. Cohen-Tannoudji, J. Dupont-Roc, G. Grynberg, AtomPhotons Interactions (Wiley, New-York, 1991), p. 385

17. C.W. Gardiner, Handbook of Stochastic Methods (Springer Verlag, Berlin, 1985); H.J. Carmichael, Phys. Rev. A 33, 3262 (1986)

18. J. Söding, D. Guéry-Odelin, P. Desbiolles, G. Ferrari, J. Dalibard, Phys. Rev. Lett. 80, 1869 (1998)

19. M. Kitawaga, M. Ueda, Phys. Rev. A 47, 5138 (1993); for our system, in which the number of atoms is much greater than unity, this definition of the squeezing parameter is consistent with that given in reference [1]. 